\title{
Nonhyperbolic one-dimensional invariant sets with a countably infinite collection of inhomogeneities
}

\author{
by
}

\author{
Chris Good (Birmingham), Robin Knight (Oxford) \\ and Brian Raines (Waco, TX)
}

\begin{abstract}
We examine the structure of countable closed invariant sets under a dynamical system on a compact metric space. We are motivated by a desire to understand the possible structures of inhomogeneities in one-dimensional nonhyperbolic sets (inverse limits of finite graphs), particularly when those inhomogeneities form a countable set. Using tools from descriptive set theory we prove a surprising restriction on the topological structure of these invariant sets if the map satisfies a weak repelling or attracting condition. We show that for a family of conceptual models for the Hénon attractor, inverse limits of tent maps, these restrictions characterize the structure of inhomogeneities. We end with several results regarding the collection of parameters that generate such spaces.
\end{abstract}

1. Introduction. R. F. Williams showed that one-dimensional hyperbolic attractors can be realized as inverse limits of one-dimensional branched manifolds [18]. He extended this result to higher dimensions in [19]. The bonding maps used in his construction had a certain amount of regularity that ensured the resulting space was a hyperbolic attractor. Namely in Williams' construction branch-points are mapped to other branch-points and edges are mapped monotonically onto unions of edges. This forces the space to be locally the product of a Cantor set and an arc.

These hyperbolic spaces, and their higher dimensional analogues, have been the focus of much study in the last few years. Notably they have arisen in the study of substitution tiling spaces (cf. [1] for $n$-dimensional tilings and [5] for 1-dimensional tilings). Sadun and Williams have recently shown that $n$-dimensional tiling spaces are indeed Cantor set fiber bundles just like the hyperbolic attractors described above [17]. In 2001 M. Barge and B. Diamond utilized a "pattern" that arose from the regular struc-

2000 Mathematics Subject Classification: 37B45, 37E05, 54F15, 54H20.

Key words and phrases: attractor, invariant set, inverse limits, unimodal, continuum, indecomposable. 
ture inherent in Williams' construction [18] to characterize one-dimensional hyperbolic attractors that are orientable and one-dimensional substitution tiling spaces [4]. I. Yi has also used Williams' description to find topological invariants for the nonorientable case [20].

Unlike the hyperbolic case in which each point has a neighborhood homeomorphic to the product of a Cantor set and an open arc, in a onedimensional nonhyperbolic space there are many inhomogeneities, i.e. points that have neighborhoods containing branch-points, endpoints, "folded up" arcs or even small non-arclike pieces (cf. [15] and [16]).

In this paper we continue our investigation of one-dimensional invariant sets that are nonhyperbolic [16]. We consider inverse limits of maps of finite graphs. We do not impose the condition that branch-points are mapped to branch-points or that edges are mapped onto a union of edges. Rather the spaces under consideration possess the property of the Hénon attractor discussed by Barge, Brucks and Diamond in [2]. Namely, neighborhoods of points in these spaces appear at first glance to have a nice regular structure, such as being the product of a Cantor set and an open arc, but upon closer scrutiny, one sees that many neighborhoods contain collections of arcs that are hopelessly folded up. Arc components do not travel "straight" around the attractor, rather they turn around and fold in complicated ways throughout the space (cf. [10, p. 268]).

In [16] we demonstrate that the points with neighborhoods that are not homeomorphs of the product of a Cantor set and an open arc fall into three categories:

(1) branch-points or limits of arbitrarily small non-arclike pieces,

(2) endpoints or limits of endpoints of some small arclike pieces,

(3) limits of "folded" arcs.

It follows quickly from the precise definitions of the preceding three classes of points that each defining property is topological. Moreover, we showed that points in these sets correspond to points that always project into certain $\omega$-limit sets. This implies that the topological structure of these inhomogeneities is closely related to the topological and dynamical structure of the relevant $\omega$-limit sets.

In the next section we discuss in more detail the precise structure of inhomogeneities in nonhyperbolic invariant sets. Then, in $\S 3$, we prove one of the main results of the paper. Specifically we prove the surprising restriction that the limit type of the collection of inhomogeneities is necessarily 0,1 or $\alpha+2$ for some countable ordinal $\alpha$ (Corollary 3.6). The next few sections of the paper are devoted to demonstrating that this restriction is sufficient in the context of inverse limits of tent map cores. We give the important preliminaries from descriptive set theory and dynamics in $\S 4$ and $\S 5$. Then 
in $\S 6$, given a countable ordinal $\alpha$, we construct a tent map core with the property that the inhomogeneities in its inverse limit space have limit type $\alpha+2$, thus demonstrating the sufficiency of the restriction given in Corollary 3.6. In $\S 7$ we define the folding spectrum, an ordered pair with first coordinate a countable ordinal and second coordinate a positive integer, of a nonhyperbolic invariant set, and we demonstrate that it is topological. From this definition and the examples constructed in $\S 6$ we prove that there are uncountably many (actually $\omega_{1}$ many) nonhomeomorphic inverse limits induced by tent map cores (Theorem 7.9). Finally, in $\S 8$ we show that for a given folding spectrum $(\alpha, n)$, there is a dense set of parameters of size $\mathfrak{c}$ in $[\sqrt{2}, 2]$ that generate a tent map core with inverse limit space having folding spectrum $(\alpha, n)$ (Theorems 8.1 \& 8.2).

2. Inhomogeneities in nonhyperbolic invariant sets. We encourage the reader unfamiliar with techniques from the theory of inverse limit spaces to see [11] or [12].

In this section we will mention many of the preliminary definitions and results regarding inhomogeneities in one-dimensional nonhyperbolic invariant sets. For a more detailed discussion see [15] or [16]. We consider a nonhyperbolic invariant set to be an inverse limit on a finite graph. Let $G$ be a graph and let $f: G \rightarrow G$ be a map. We denote the inverse limit of $f$ on $G$ by $\lim _{G} G f$, and we denote the $n$th projection map restricted to the inverse limit space by simply $\pi_{n}$. All one-dimensional compact connected metric spaces are realizable as such spaces, although not necessarily with one bonding map or factor space. However, the class of topological spaces we are considering is quite large.

In order to guarantee that the action of the induced map on arcs in the inverse limit space is expanding, we usually assume that $f$ is locally eventually onto, l.e.o., i.e. for every compact connected $B \subset G$ there is a positive integer $n$ such that $f^{n}(B)=G$.

If $f$ is a function, $U$ is a set, and $\mathcal{U}$ is a collection of sets, we will abuse notation throughout the paper by using $f(U)$ to mean the collection of points $\{f(x): x \in U\}$ and $f(\mathcal{U})=\{f(U): U \in \mathcal{U}\}$. We define the mesh of $\mathcal{U}$, mesh $(\mathcal{U})$, to be the largest diameter of its elements, provided such a number exists. The $\omega$-limit set of a point $x$ is defined as

$$
\omega(x)=\bigcap_{n \in \mathbb{N}} \overline{\left\{f^{m}(x): m \geq n\right\}},
$$

and the $\omega$-limit set of a set $A$ as

$$
\omega(A)=\bigcup_{x \in A} \omega(x) .
$$


We denote the closure of a set $A$ by $\bar{A}$. We call a compact, connected, metric space a continuum, and a compact connected subset of a continuum a subcontinuum.

Let $X$ be a topological space and $x \in X$. Let $V$ be an open set containing $x$. Call a finite collection $\mathcal{U}=\left\{U_{1}, \ldots, U_{n}\right\}$ of open subsets of $V$ a linear cover or simple chain provided $\bar{U}_{i} \cap \bar{U}_{j} \neq \emptyset$ if, and only if, $|i-j|<2$. We will call the elements of such a linear cover links. If $\operatorname{mesh}(\mathcal{U})<\varepsilon$ then we call $\mathcal{U}$ a linear $\varepsilon$-cover. Call a finite collection of linear covers of $V, \mathcal{U}=\left\{\mathcal{U}_{1}, \ldots, \mathcal{U}_{m}\right\}$ where $\mathcal{U}_{i}=\left\{U_{1}^{i}, \ldots, U_{p_{i}}^{i}\right\}$, a local chaining of $V$ if $\bigcup_{i \leq m} \mathcal{U}_{i}$ covers $V$ and $\overline{U_{j}^{i}} \cap \overline{U_{l}^{k}} \neq \emptyset$ if, and only if, $i=k$ and $|j-l|<2$. Call each element $\mathcal{U}_{i}$ of a local chaining $\mathcal{U}$ of $V$ a strand of $\mathcal{U}$. If each strand of $\mathcal{U}$ is a linear $\varepsilon$-cover, call $\mathcal{U}$ a local $\varepsilon$-chaining of $V$.

Let $\mathcal{C}=\left\{C_{1}, \ldots, C_{n}\right\}$ and $\mathcal{D}=\left\{D_{1}, \ldots, D_{m}\right\}$ be linear covers such that for each $i \leq m$ there is a $j \leq n$ such that $\bar{D}_{i} \subseteq C_{j}$. Then we say $\mathcal{D}$ refines $\mathcal{C}$ and we write $\mathcal{D} \leq \mathcal{C}$.

If $\mathcal{U}=\left\{\mathcal{U}_{1}, \ldots, \mathcal{U}_{n}\right\}$ and $\mathcal{V}=\left\{\mathcal{V}_{1}, \ldots, \mathcal{V}_{m}\right\}$ are local chainings of $V$ with the property that

(1) every strand of $\mathcal{V}$ refines exactly one strand of $\mathcal{U}$,

(2) every strand of $\mathcal{U}$ is refined by some strand of $\mathcal{V}$,

then we say $\mathcal{V}$ refines $\mathcal{U}$ and we write $\mathcal{V} \leq \mathcal{U}$. The mesh of a local chaining is the largest mesh of its strands.

$X$ is locally chainable at $x$ iff there is a neighborhood $U$ of $x$ and a sequence $\left\{\mathcal{C}_{i}\right\}_{i=1}^{\infty}$ of local chainings of $U$ such that

(1) $\operatorname{mesh}\left(\mathcal{C}_{i}\right) \rightarrow 0$ as $i \rightarrow \infty$,

(2) $\mathcal{C}_{i} \leq \mathcal{C}_{i-1}$

We will say that the neighborhood $U$ is locally chainable in this case. The notion of local chainability appeared earlier in [14].

Let $\mathcal{U}$ be a local chaining of some $U \subseteq X$. Let $\mathcal{L}=\left\{L_{1}, \ldots, L_{p}\right\}$ be a linear cover that refines some strand $\mathcal{U}_{i}$ of $\mathcal{U}$. Let $L$ be a link of $\mathcal{U}_{i}$. We say that $\mathcal{L}$ turns in $L$ provided there is a link $M$ in $\mathcal{U}_{i}$, adjacent to $L$, and integers $a$ and $b$ with $1 \leq a<b-1<b \leq p$ such that

(1) $L_{a}, L_{b} \subseteq M$,

(2) $L_{j} \subseteq L-M$ for some $a<j<b$,

(3) $\bigcup_{i=a}^{b} L_{i} \subseteq L \cup M$.

We call $L$ a local turnlink, or just a turnlink. If every local $\varepsilon$-chaining of $U$ that refines $\mathcal{U}_{i}$ has a turnlink in $L$ then we call $L$ a local essential turnlink, or just an essential turnlink.

Let $X$ be a metric space with a point $x \in X$ such that $X$ is locally chainable at $x$. Let $U$ be a neighborhood of $x$ that is locally chainable. Call 
$x$ a folding point of $X$ if for every $\varepsilon>0$ there is a local $\varepsilon$-chaining $\mathcal{C}$ of $U$ that contains $x$ in an essential turnlink. Denote the set of folding points for a space $X$ by $\mathbb{F d}(X)$.

Let $G$ be a finite graph and let $f: G \rightarrow G$ be a continuous l.e.o. map with finitely many turning points. Let $X_{G}=\varliminf\{G, f\}$. Let $V$ be the set of branch-points of $G$, and let $C$ be the set of turning points for $f$. In [16, Theorem $2.1 \&$ Lemma 4.1] we showed that the set of folding points of $X_{G}$ contains all of the points $x \in X_{G}$ that are locally chainable and do not have neighborhoods homeomorphic to the product of a Cantor set and an arc. So define the set

$$
\operatorname{In}\left(X_{G}\right)=\mathbb{F} \mathrm{d}\left(X_{G}\right) \cup\left\{x \in X_{G}: X_{G} \text { is not locally chainable at } x\right\} .
$$

Thus if $x \in X_{G} \backslash \operatorname{In}\left(X_{G}\right)$ then $x$ has a neighborhood homeomorphic to the product of a Cantor set and an arc.

We also proved several theorems that connect the set $\operatorname{In}\left(X_{G}\right)$ with the $\omega$-limit sets of the turning points for $f$ and the branch-points of $G$. For completeness we now state the relevant theorems.

Theorem 2.1 ([16, Theorem 3.6]). Let $x \in X_{G}$ be such that $\pi_{n}(x)=$ $x_{n} \notin \omega(V)$ for all $n \in \mathbb{N}$. Then $X_{G}$ is locally chainable at $x$.

Theorem $2.2\left(\left[16\right.\right.$, Theorem 3.7]). Let $x \in X_{G}$ be such that if $\pi_{n}(x)=$ $x_{n} \in \omega(V)$ then the set $V_{n}=\left\{v_{j_{1}}, \ldots, v_{j_{r}}: x_{n} \in \omega\left(v_{j_{i}}\right)\right\}$ has the property that $V_{n} \cap \omega(V)=\emptyset$. Then $X_{G}$ is locally chainable at $x$.

Theorem 2.3 ([16, Corollary 4.8(1)]). Let $x \in X_{G}$ be a point of local chainability. Then $x$ is a folding point for $X_{G}$ if, and only if, $\pi_{n}(x)=x_{n} \in$ $\omega(C)$ for all $n \in \mathbb{N}$.

In light of these theorems and the fact that $f[\omega(A)]=\omega(A)$ for all sets $A$, we see that to analyze the structure of the set $\operatorname{In}\left(X_{G}\right)$ we need to analyze the structure of $\omega(C)$ and $\omega(V)$.

3. Restriction on scattered height. In this section we assume that $X_{G}$ is an inverse limit on a finite graph $G$ with bonding map $f$ that is l.e.o. and has finitely many turning points. We also assume that the set of inhomogeneities is countably infinite. Since $\operatorname{In}\left(X_{G}\right)$ is the collection of points that do not have a neighborhood homeomorphic to the product of a Cantor set and an open arc, the set $\operatorname{In}\left(X_{G}\right)=\mathbb{F} \mathrm{d}\left(X_{G}\right) \cup\left\{x \in X_{G}\right.$ : $X_{G}$ is not locally chainable at $\left.x\right\}$ is the complement of a collection of open neighborhoods and as such it is closed. In fact it is compact.

We begin this section with a brief discussion of the topological structure of countable, compact Hausdorff spaces. 
The Cantor-Bendixson derivative $A^{\prime}$ of a subset $A$ of a space $X$ is the set of limit points of the set $A$, and the iterated Cantor-Bendixson derivatives of the space $X$ are defined inductively by

$$
\begin{aligned}
X^{(0)} & =X, \\
X^{(\alpha+1)} & =\left(X^{(\alpha)}\right)^{\prime}, \\
X^{(\lambda)} & =\bigcap_{\alpha<\lambda} X^{(\alpha)} \quad \text { if } \lambda \text { is a limit ordinal. }
\end{aligned}
$$

Clearly for some ordinal $\gamma, X^{(\gamma)}=X^{(\gamma+1)}$, and $X$ is said to be scattered if this set is empty and $X$ is nonempty. In this case, a point of $X$ has a well-defined rank, often called the scattered height or limit type of $x$, defined by $\operatorname{lt}(x)=\alpha$ if and only if $x \in X^{(\alpha)} \backslash X^{(\alpha+1)}$. The $\alpha$ th level $L_{\alpha}$ of $X$ (or, more formally, $L_{\alpha}^{X}$ ) is then the set of all points of limit type $\alpha$. Clearly $L_{\alpha}$ is the set of isolated points of $X^{(\alpha)}$. The limit type (or scattered height) $\operatorname{Lt}(X)$ of the space $X$ itself is the least ordinal $\gamma$ such that $X^{(\gamma)}=\emptyset$. Notice that $\operatorname{Lt}(X)$ is the Cantor-Bendixson rank of $X$ (see [13, p. 33]). If $X$ is a compact scattered space, then $\operatorname{Lt}(X)$ is a successor ordinal $\alpha+1$ and the level $L_{\alpha}$ is finite.

If an ordinal (regarded as the set of its own predecessors) is given its natural order topology then it forms a scattered space, and it is a standard topological fact that every countable, compact Hausdorff space is not only scattered but homeomorphic to a countable successor ordinal. Moreover, every successor (i.e. compact) ordinal $\alpha$ has a canonical decomposition, the Cantor normal form, $\alpha=\sum_{i=1}^{k} \omega^{\delta_{i}} n_{i}$, where $n_{i}$ is a positive integer and $\delta_{i+1}<\delta_{i}$. Hence every countable, compact Hausdorff space of given height $\gamma$ is homeomorphic to a finite number of disjoint copies of the least ordinal space $\omega^{\gamma}$ of limit type $\gamma$.

In this section we prove a surprising restriction on the possible limit type of the set of inhomogeneities of $X_{G}$ assuming that $\operatorname{In}\left(X_{G}\right)$ is countable. The main result of this section, Corollary 3.6, states that if $\operatorname{In}\left(X_{G}\right)$ is countable, then $\operatorname{Lt}\left[\operatorname{In}\left(X_{G}\right)\right]$ is actually $\alpha+2$ for some ordinal $\alpha$. This shows, for instance, that there is no space $X_{G}$ with $\operatorname{Lt}\left[\operatorname{In}\left(X_{G}\right)\right]=\omega$ or with $\operatorname{Lt}\left[\operatorname{In}\left(X_{G}\right)\right]=\omega+1$. We accomplish this by examining the possible limit types of periodic points contained in $\operatorname{In}\left(X_{G}\right)$. Since

$$
h\left[\operatorname{In}\left(X_{G}\right)\right]=\mathbb{I n}\left(X_{G}\right)
$$

for every homeomorphism $h: X_{G} \rightarrow X_{G}$, it is not hard to see that the "top" level of $\operatorname{In}\left(X_{G}\right)$ must be finite and consist of periodic points for $h$. By showing that the only limit types available for periodic points in $\operatorname{In}\left(X_{G}\right)$ are successor ordinals, we show that $\operatorname{Lt}\left[\operatorname{In}\left(X_{G}\right)\right]$ is a successor of a successor.

We begin by considering the problem in a more general context. 
Lemma 3.1. Let $X$ be a compact metric space, let $f: X \rightarrow X$ be a continuous finite-to-one map, and let $A$ be a closed subset of $X$ with $f(A)=A$. Then $f\left(L_{\alpha}^{A}\right) \supseteq L_{\alpha}^{A}$ for all countable ordinals $\alpha$.

Proof. Since $f$ maps $A$ onto itself, in particular $f\left(L_{0}^{A}\right) \supseteq L_{0}^{A}$. Let $\alpha$ be a countable ordinal and suppose that $f\left(L_{\alpha}^{A}\right) \supseteq L_{\alpha}^{A}$. Let $z \in L_{\alpha+1}^{A}$. Then there is a sequence $\left(y_{n}\right)_{n \in \mathbb{N}}$ of points in $L_{\alpha}^{A}$ converging to $z$. For each $n$, let $y_{n}^{\prime}$ be a preimage of $y_{n}$ in $L_{\alpha}^{A}$. Let $\left(y_{n_{i}}^{\prime}\right)_{i \in \mathbb{N}}$ be a convergent subsequence of $\left(y_{n}^{\prime}\right)_{n \in \mathbb{N}}$. Let $z^{\prime}=\lim _{i \rightarrow \infty} y_{n_{i}}^{\prime}$. Then $\operatorname{lt}\left(z^{\prime}\right)>\alpha$. Since $f$ is continuous, $f\left(z^{\prime}\right)=z$. Thus $\operatorname{lt}\left(z^{\prime}\right) \leq \alpha+1$. Hence $\operatorname{lt}\left(z^{\prime}\right)=\alpha+1$. This implies that $f\left(L_{\alpha+1}^{A}\right) \supseteq L_{\alpha+1}^{A}$.

Now suppose that $\alpha$ is a limit and the theorem is true for all $\beta<\alpha$. Then if $z \in L_{\alpha}^{A}$ with $y_{n} \in L_{\beta_{n}}^{A}$ and $\beta_{n} \rightarrow \alpha$ as $n \rightarrow \infty$ the above argument will provide a preimage $z^{\prime}$ of $z$ with $\operatorname{lt}\left(z^{\prime}\right)>\beta$ for all $\beta<\alpha$. So $\operatorname{lt}\left(z^{\prime}\right) \geq \alpha$, but since $f\left(z^{\prime}\right)=z, \operatorname{lt}\left(z^{\prime}\right)=\alpha$. This completes the proof.

Thus, by the previous lemma, for every $\alpha$ we have

$$
h\left[L_{\alpha}^{\operatorname{In}\left(X_{G}\right)}\right]=L_{\alpha}^{\mathbb{I n}\left(X_{G}\right)}
$$

for every homeomorphism $h: X_{G} \rightarrow X_{G}$. Hence if $\operatorname{Lt}\left[\operatorname{In}\left(X_{G}\right)\right]=\gamma+1$, then $L_{\gamma}^{\mathbb{I n}\left(X_{G}\right)}$ is a finite set that is preserved by every homeomorphism of $X_{G}$, so it consists of finitely many periodic orbits for each such homeomorphism. Let $\widehat{f}$ denote the induced homeomorphism on $X_{G}$, i.e. the shift homeomorphism induced by the bonding map $f$. By our assumptions about the bonding map $f$, we know that $\widehat{f}$ stretches small arcs in $X_{G}$.

Given a compact metric space $X$, a self-map $f$ of $X$, a positive number $\varepsilon$, a periodic point $x$ of $f$ of period $r$, and a set $A \subseteq X$, we say that $x$ is nearly $\varepsilon$-repelling with respect to $A$ provided that if $\left(z_{n}\right)_{n \in \mathbb{N}} \subseteq A$ converges to $x$ then

(*) there is a subsequence $\left(z_{n_{i}}\right)_{i \in \mathbb{N}}$ such that for each $i \in \mathbb{N}$, there is some positive integer $m_{i}$ such that $d\left(f^{r m_{i}}\left(z_{n_{i}}\right), x\right) \geq \varepsilon$.

The main result of this section relies on the fact that each point in the top level of $\operatorname{In}\left(X_{G}\right)$ is a periodic point that is nearly $\varepsilon$-repelling with respect to $\operatorname{In}\left(X_{G}\right)$ under either $\widehat{f}$ or $\widehat{f}^{-1}$.

Lemma 3.2. Let $X$ be a compact metric space, $f$ a self-map of $X, x$ a periodic point of $f$ of period $r$, and $\left(z_{n}\right)_{n \in \mathbb{N}}$ a sequence of points converging to $x$. Suppose that for some $k \in \mathbb{N}, x$ is nearly $1 / 2^{k}$-repelling with respect to $\left(z_{n}\right)_{n \in \mathbb{N}}$. Let $\left(z_{n_{i}}\right)_{i \in \mathbb{N}}$ be the subsequence of $\left(z_{n}\right)_{n \in \mathbb{N}}$ guaranteed by $(*)$. Then, for each $m \geq k$, either

(1) $\left\{f^{r j}\left(z_{n_{i}}\right): j, i \in \mathbb{N}, d\left(f^{r j}\left(z_{n_{i}}\right), x\right)<1 / 2^{m} \leq d\left(f^{r j+1}\left(z_{n_{i}}\right), x\right)\right\}$ is a finite set, or 
(2) there is a point $y_{m}$ such that

(a) $0<d\left(y_{m}, x\right) \leq 1 / 2^{m}$ for each $m$,

(b) $y_{m}$ is a limit point of $\left\{z_{i}^{\prime}: z_{i}^{\prime}=f^{r_{i}}\left(z_{n_{i}}\right)\right.$ for some $\left.r_{i} \in \mathbb{N}\right\}$,

(c) if, moreover, $\operatorname{lt}\left(z_{n_{i}}\right) \geq \alpha_{i}$ for each $i \in \mathbb{N}$, then

$$
\operatorname{lt}\left(y_{m}\right) \geq \sup \left\{\alpha_{i}+1: n \in \mathbb{N}\right\} .
$$

Proof. By considering the map $f^{r}$ instead of $f$ if necessary, without loss of generality we may assume that $r=1$ and $x$ is a fixed point of $f$.

Since $\left(z_{n_{i}}\right)_{i \in \mathbb{N}}$ converges to $x$ and each $z_{n_{i}}$ is eventually mapped farther than $1 / 2^{k}$ from $x$, for each $k \leq m$, if $d\left(z_{n_{i}}, x\right)<1 / 2^{m}$, then there is some $j$ such that $d\left(f^{j}\left(z_{n_{i}}\right), x\right)<1 / 2^{m} \leq d\left(f^{j+1}\left(z_{n_{i}}\right), x\right)$. Since $X$ is compact, the set $\left\{f^{j}\left(z_{n_{i}}\right): j, i \in \mathbb{N}, d\left(f^{j}\left(z_{n_{i}}\right), x\right)<1 / 2^{m} \leq d\left(f^{j+1}\left(z_{n_{i}}\right), x\right)\right\}$ is either finite or has a limit point $y_{m}$ and $d\left(y_{m}, x\right) \leq 1 / 2^{m}$. Since $d\left(f^{j_{m, i}+1}\left(z_{n_{i}}\right), x\right) \geq 1 / 2^{m}$ for each $i \in \mathbb{N}, d\left(f\left(y_{m}\right), x\right) \geq 1 / 2^{m}$ and so $y_{m} \neq x$.

If $\operatorname{lt}\left(z_{n_{i}}\right) \geq \alpha_{i}$, then $\operatorname{lt}\left(f^{j}\left(z_{n_{i}}\right)\right) \geq \alpha_{i}$ by continuity. Hence $\operatorname{lt}(y) \geq$ $\sup \left\{\alpha_{i}+1: n \in \mathbb{N}\right\}$.

Given a compact metric space $X$, a continuous map $f$ of $X$, and a countable closed subset $A$ of $X$ that is forward invariant with respect to $f$, we now show that any periodic point in $A$ that is nearly $\varepsilon$-repelling with respect to $A$ must have successor limit type with respect to $A$.

ThEOREM 3.3. Let $X$ be a compact metric space, and $f: X \rightarrow X$ be continuous. Let $A \subset X$ be countable, closed and forward invariant with respect to $f$. Let $x$ be a periodic point of $f$ of period $r$. If $x$ is nearly $\varepsilon$-repelling with respect to $A$ and $\operatorname{lt}_{A}(x)=\alpha$, then $\alpha$ is not a limit ordinal.

Proof. Again, we may assume that $x$ is a fixed point of $f$.

Suppose that $\operatorname{lt}(x)=\alpha$ is a limit ordinal. Then there is a sequence $\left(z_{n}\right)_{n \in \mathbb{N}}$ of points in $A$ converging to $x$ such that $\operatorname{lt}\left(z_{n}\right)=\alpha_{n}$, where $\left(\alpha_{n}\right)_{n \in \mathbb{N}}$ is a strictly increasing sequence of ordinals converging to $\alpha$. Choose a subsequence $\left(z_{n_{i}}\right)_{i \in \mathbb{N}}$ that satisfies $(*)$ and notice that since $\left(\alpha_{n}\right)_{n \in \mathbb{N}}$ is an increasing sequence of ordinals, $\left(\alpha_{n_{i}}\right)_{i \in \mathbb{N}}$ is also an increasing sequence of ordinals with the same limit $\alpha$. So Lemma 3.2 applies. Since $\left(\alpha_{n_{i}}\right)_{i \in \mathbb{N}}$ is strictly increasing with supremum $\alpha$, for each $m \geq k$, either the set $\left\{f^{j}\left(z_{n_{i}}\right)\right.$ : $\left.j, i \in \mathbb{N}, d\left(f^{j}\left(z_{n_{i}}\right), x\right)<1 / 2^{m} \leq d\left(f^{j+1}\left(z_{n_{i}}\right), x\right)\right\}$ is infinite or $\left\{\operatorname{lt}\left(f^{j}\left(z_{n_{i}}\right)\right)\right.$ : $\left.j, i \in \mathbb{N}, d\left(f^{j}\left(z_{n_{i}}\right), x\right)<1 / 2^{m} \leq d\left(f^{j+1}\left(z_{n_{i}}\right), x\right)\right\}$ is a finite set containing some ordinal $\alpha^{\prime} \geq \alpha$. In either case, there is a point $y_{m}$ such that $0<d\left(y_{m}, x\right) \leq 1 / 2^{m}$ and $\operatorname{lt}\left(y_{m}\right) \geq \alpha$. Since $\left(y_{m}\right)_{m \in \mathbb{N}}$ converges to $x$ we have a contradiction and the limit type of $x$ is either 0 or a successor.

This immediately implies the following.

Corollary 3.4. Let $X$ be a compact metric space and let $f$ be a selfmap of $X$ with repelling periodic point $x$. Then $\operatorname{lt}_{A}(x)$ is not a limit ordinal for any closed, forward invariant, countable set $A$. 
Proof. A repelling periodic point is easily seen to be nearly $\varepsilon$-repelling with respect to any closed, forward invariant, countable set $A$.

In $X_{G}$, however, points that are periodic under $\widehat{f}$ are not necessarily repelling under $\widehat{f}$. But they are nearly $\varepsilon$-repelling with respect to $\operatorname{In}\left(X_{G}\right)$. This gives us the main result of the section. We write $\operatorname{lt}(x)$ for $\operatorname{lt}_{\mathbb{I n}\left(X_{G}\right)}(x)$.

TheOREM 3.5. Let $x \in \operatorname{In}\left(X_{G}\right)$ be a periodic point under the shift homeomorphism $\widehat{f}$. Then $\operatorname{lt}(x)$ is not a limit ordinal.

Proof. Without loss of generality assume that $x$ is fixed under the shift homeomorphism and $x=(\bar{x}, \bar{x}, \ldots)$.

Suppose that $\operatorname{lt}(x)=\alpha$ and that $\left(z_{n}\right)_{n \in \mathbb{N}}$ is a sequence from $\operatorname{In}\left(X_{G}\right)$ converging to $x$ such that $\alpha=\sup \left\{\operatorname{lt}\left(z_{n}\right)+1: n \in \mathbb{N}\right\}$. We will show that for either the shift homeomorphism $\widehat{f}\left[\left(x_{1}, x_{2}, \ldots\right)\right]=\left(f\left(x_{1}\right), x_{1} \ldots\right)$, or its inverse $\widetilde{f}\left[\left(x_{1}, x_{2}, \ldots\right)\right]=\left(x_{2}, x_{3}, \ldots\right)$, there is a subsequence $\left(z_{n_{i}}\right)_{i \in \mathbb{N}}$ of $\left(z_{n}\right)_{n \in \mathbb{N}}$ that satisfies $(*)$ and has the property that $\alpha=\sup \left\{\operatorname{lt}\left(z_{n_{i}}\right)+1: i \in \mathbb{N}\right\}$. The result will follow.

Let $\lambda>0$ be small enough so that $|\bar{x}-w| \geq \lambda$ for each $w \in f^{-1}(\bar{x})$. Let $k \in \mathbb{N}$ be large enough so that $1 / 2^{k+1}<\lambda$ and $B_{1 / 2^{k+1}}(\bar{x})$ is contained in the basin of repulsion for $\bar{x}$ (recall that $f$ is l.e.o. so each point is a repellor).

We begin by assuming that there is a subsequence $\left(z_{n_{i}}\right)_{i \in \mathbb{N}}$ of $\left(z_{n}\right)_{n \in \mathbb{N}}$ with $\alpha=\sup \left\{\operatorname{lt}\left(z_{n_{i}}\right)+1: i \in \mathbb{N}\right\}$ and $\pi_{1}\left(z_{n_{i}}\right)=\bar{x}$ for each $i \in \mathbb{N}$. Let $m_{i}$ be the least integer $j$ such that $\pi_{j}\left(z_{n_{i}}\right) \neq \bar{x}$. Clearly $\pi_{m_{i}}\left(z_{n_{i}}\right) \in f^{-1}(\bar{x})$, so $\left|\pi_{m_{i}}\left(z_{n_{i}}\right)-\bar{x}\right| \geq \lambda$. Then $d\left(\widetilde{f}^{m_{i}}\left(z_{n_{i}}\right), x\right) \geq \lambda / 2 \geq 1 / 2^{k}$. Hence $\left(z_{n_{i}}\right)_{i \in \mathbb{N}}$ satisfies $(*)$.

Now assume that there is no such subsequence. So either there is a tail $\left(z_{n}\right)_{n \geq M}$ of $\left(z_{n}\right)_{n \in \mathbb{N}}$ such that $\alpha=\sup \left\{\operatorname{lt}\left(z_{n}\right)+1: n \geq M\right\}$ and $\pi_{1}\left(z_{n}\right) \neq \bar{x}$, or $\alpha$ is not a limit ordinal. In the first case, consider $z_{n}$. If $\pi_{1}\left(z_{n}\right) \notin B_{1 / 2^{k+1}}(\bar{x})$ then clearly there is an integer, namely $m_{n}=0$, such that $d\left(\widehat{f}^{m_{n}}\left(z_{n}\right), x\right) \geq$ $1 / 2^{k}$. On the other hand, if $\pi_{1}\left(z_{n}\right) \in B_{1 / 2^{k+1}}(\bar{x}) \backslash\{\bar{x}\}$, then clearly there is an integer $m_{n}$ so that $f^{m_{n}}\left[\pi_{1}\left(z_{n}\right)\right] \notin B_{1 / 2^{k+1}}(\bar{x})$. Either way $d\left(\widehat{f}^{m_{n}}\left(z_{n}\right), \bar{x}\right)$ $\geq 1 / 2^{k}$ and $\left(z_{n}\right)_{n \geq M}$ satisfies $(*)$.

Corollary 3.6. If $\operatorname{In}\left(X_{G}\right)$ is countable, then $\operatorname{Lt}\left[\operatorname{In}\left(X_{G}\right)\right]$ is either 0,1 , or $\alpha+2$ for some countable ordinal $\alpha$.

Proof. Let $\operatorname{Lt}\left[\operatorname{In}\left(X_{G}\right)\right]=\beta+1$, where $\beta$ is a countable ordinal. Then $L_{\beta}^{\operatorname{In}\left(X_{G}\right)}$ is a collection of periodic points under the map $\widehat{f}$ of scattered height $\beta$. Let $x$ be such a point with period $n$. By Theorem 3.5, $\beta$ cannot be a limit ordinal. Hence $\operatorname{lt}(z)=\beta=\alpha+1$ for some $\alpha$, and so $\beta+1=\alpha+2$. This establishes the corollary. 
We also have the following restriction for $\omega$-limit sets of continuous maps of the interval:

COROLlary 3.7. Let $f$ be a map of $[0,1]$ such that every periodic point of $f$ is repelling. Let $x \in[0,1]$ and suppose that $\omega(x)$ is countably infinite. If $z \in \omega(x)$ is a periodic point, then $\operatorname{lt}(z)$ is 0 or a successor. Moreover, $\operatorname{Lt}(\omega(x))$ is 0,1 or $\alpha+2$ for some $\alpha \in \omega_{1}$.

Proof. Since every periodic point of $f$ is repelling, by Theorem 3.3 the limit type of a periodic point $z$ of $\omega(c)$ is not a limit ordinal. Since $\omega(c)$ is a countable compact set, $T=L_{\beta}^{\omega(c)}$ is finite for some $\beta \in \omega_{1}$. Since $f(T)=T, T$ consists of periodic points and $\beta=\alpha+1$ is a successor. Hence $\operatorname{Lt}(\omega(c))=\alpha+2$ for some $\alpha \in \omega_{1}$.

It is an easy exercise to alter the previous proofs and use the inverse of the map if we assume that the action of the map is nearly $\varepsilon$-attracting rather than nearly $\varepsilon$-repelling on the invariant set.

4. Preliminaries from descriptive set theory. In the previous section we showed that if the set $\operatorname{In}\left(X_{G}\right)$ is countable then it must have scattered height 0,1 or $\alpha+2$ for some countable ordinal $\alpha$. The next obvious question is: "For each countable ordinal $\alpha$, does there exist a nonhyperbolic invariant set $X_{G}$ with the property that $\operatorname{In}\left(X_{G}\right)$ has scattered height exactly $\alpha+2$ ?" In the next few sections we answer this question in the affirmative. We do this by considering simpler inverse limit spaces: inverse limits of unimodal maps of the interval $[0,1]$. We showed in [16] that, given a unimodal map $f:[0,1] \rightarrow[0,1]$ with critical point $c$, the set $\operatorname{In}(\stackrel{\lim }{\longleftarrow}\{[0,1], f\})$ coincides exactly with the set of points in $\lim \{[0,1], f\}$ that always project into $\omega(c)$. So, in order to construct a space with inhomogeneities that have scattered height $\alpha+2$, we will construct a unimodal map (actually a tent map) with critical point $c$ with the property that $\omega(c)$ has scattered height $\alpha+2$.

In this section we briefly describe for completeness the construction of a well-founded tree of height $\alpha$ for each countable ordinal $\alpha$. Such trees have the remarkable property that they are countable with finite branches but can have height $\alpha$ for any countable ordinal $\alpha$. For more details we refer the reader to $[13, \mathrm{I} .2]$.

Let $\mathcal{A}$ be a countably infinite set of symbols and let $\mathcal{A}^{<\mathbb{N}}$ be the set of all finite sequences of elements of $\mathcal{A}$. Given two elements $s, t \in \mathcal{A}^{<\mathbb{N}}$ we say that $t \triangleleft s$ if $s$ is an initial segment of $t$, i.e. $t=\left(t_{1}, \ldots, t_{n}\right)$ and $s=\left(t_{1}, \ldots, t_{m}\right)$ for some $m<n$. If $n=m+1$, then $t$ extends $s$ by one symbol and we write $t \lessdot s$. If $s=\left(s_{1}, \ldots, s_{m}\right)$ and $t=\left(t_{1}, \ldots, t_{n}\right)$, then we denote $\left(s_{1}, \ldots, s_{m}, t_{1}, \ldots, t_{n}\right)$ by $s t$.

A subset $\mathcal{T}$ of $\mathcal{A}^{<\mathbb{N}}$ is said to be a tree on $\mathcal{A}$ if it is closed under initial segments, i.e. whenever $t \in \mathcal{T}$ and, for some $s \in \mathcal{A}^{\mathbb{N}}, t \triangleleft s$, then $s \in \mathcal{T}$. 
Since the null sequence ( ) is an initial segment of any sequence, it is the top element of every tree on $\mathcal{A}$.

An infinite branch in $\mathcal{T}$ is an infinite sequence $b=\left(b_{1}, b_{2}, b_{3}, \ldots\right)$ of elements from $\mathcal{A}$ such that $\left(b_{1}, \ldots, b_{n}\right) \in \mathcal{T}$ for all $n \in \mathbb{N}$. If $\mathcal{T}$ has no infinite branches, then the relation $\triangleleft$ is well-founded (i.e. has no infinite descending chains) and $\mathcal{T}$ is said to be a well-founded tree.

We can inductively associate a well-defined ordinal height ht $\mathcal{T}(s)$ to each element $s$ of a well-founded tree $\mathcal{T}$ by declaring

$$
\operatorname{ht}_{\mathcal{T}}(s)=\sup \left\{\operatorname{ht}_{\mathcal{T}}(t)+1: t \in \mathcal{T} \text { and } t \triangleleft s\right\},
$$

and associate to each well-founded tree $\mathcal{T}$ a well-defined height $\operatorname{Ht}(\mathcal{T})=$ $\operatorname{ht}_{\mathcal{T}}(())$. Clearly, if $t \triangleleft s$, then ht $\operatorname{ht}_{\mathcal{T}}(t)<\operatorname{ht}_{\mathcal{T}}(s)$, ht $\operatorname{Lt}_{\mathcal{T}}(())>\operatorname{ht}_{\mathcal{T}}(s)$ for any ( ) $\neq s \in \mathcal{T}$, and if $s \in \mathcal{T}$ has maximal length, then $\operatorname{ht}_{\mathcal{T}}(s)=0$.

Trees of height $\alpha$ can be defined recursively. Let $s_{a}$ be the singleton sequence $(a)$ for some $a \in \mathcal{A}$. Obviously $\mathcal{T}_{0}=\{\emptyset\}$ is a tree of height 0 on $\mathcal{A}$. So suppose that $\alpha=\beta+1$ and assume that there is a tree $\mathcal{T}_{\beta}$ on $\mathcal{A}$ of height $\beta$. Since $\mathcal{A}$ is infinite, there is, in fact, a countably infinite family $\left\{\mathcal{U}_{n}: n \in \mathbb{N}\right\}$ of disjoint trees, each order isomorphic to $\mathcal{T}_{\beta}$. Define

$$
\mathcal{T}_{\alpha}=\{()\} \cup\left\{s_{a} t: t \in \mathcal{U}_{n}, n \in \mathbb{N}\right\} .
$$

Clearly $\mathcal{T}_{\alpha}$ is a well-founded tree on $\mathcal{A}$. Moreover ht $\mathcal{T}_{\alpha}\left(s_{a} t\right)=\mathrm{ht}_{\mathcal{U}_{n}}(t)$ for every $t \in U_{n}$ and $n \in \mathbb{N}$, so $\operatorname{Ht}\left(\mathcal{T}_{\alpha}\right)=\beta+1=\alpha$.

Now suppose that $\alpha$ is a limit ordinal and that for every $\beta<\alpha$ there is a tree $\mathcal{T}_{\beta}$ of height $\beta$ on $\mathcal{A}$. Again, since $\mathcal{A}$ is countably infinite, we may assume that $\mathcal{I}_{\beta}$ and $\mathcal{T}_{\gamma}$ are disjoint whenever $\beta \neq \gamma<\alpha$. Define

$$
\mathcal{T}_{\alpha}=\{()\} \cup\left\{s_{a} t: t \in \mathcal{T}_{\beta}, \beta<\alpha\right\} .
$$

Again it is clear that $\mathcal{T}_{\alpha}$ is a well-founded tree and that $\operatorname{Ht}\left(\mathcal{T}_{\alpha}\right)=\alpha$.

Notice that, as constructed, if $t \in \mathcal{T}_{\alpha}=\mathcal{T}$ for some $\alpha$ and ht $\mathcal{T}(t)=\gamma$ then if $\gamma=\beta+1$, there are infinitely many $s \in \mathcal{T}$ such that ht $\mathcal{T}(s)=\beta$ and $s \lessdot t$, and if $\gamma$ is a limit, then for each $\beta<\gamma$, there is some $s \lessdot t$ such that $\operatorname{ht}_{\mathcal{T}}(s)=\beta$.

5. Preliminaries from dynamics of maps of the interval. We now provide the necessary definitions from dynamics for the examples that we construct in $\S 6$. Let $f:[0,1] \rightarrow[0,1]$ be a unimodal map with critical point $c$. For any $x \in[0,1]$ we define the itinerary of $x$ under $f$ to be $I_{f}(x)=t_{0} t_{1} t_{2} \ldots$ where $t_{i} \in\{0,1, C\}$ and $t_{i}=0$ if $f^{i}(x) \in[0, c), t_{i}=1$ if $f^{i}(x) \in(c, 1]$, and $t_{i}=C$ if $f^{i}(x)=c$. The kneading sequence for $f, K(f)$, is $I_{f}[f(c)]$. We adopt the standard convention of stopping an itinerary at the first occurrence of the symbol $C$. In the rest of the paper if $A$ is a finite word, $B$ is a word, and $A$ is an initial segment of $B$ we will write $A \sqsubseteq B$. 
A sequence $M$ in symbols 0 and 1 is primary provided it is not a *product, i.e. there is no finite word $W$ and sequence $\left(u_{i}\right)_{i \in \mathbb{N}}$ of points from $\{0,1\}$ with $M=W u_{1} W u_{2} W u_{3} \ldots$ The shift map $\sigma$ on sequences is defined by $\sigma\left[t_{0} t_{1} \ldots\right]=t_{1} t_{2} \ldots$ We order sequences using the parity-lexicographic ordering $\prec$. To define this order we first define $0<C<1$. Let $t=t_{0} t_{1} t_{2} \ldots$ and $s=s_{0} s_{1} s_{2} \ldots$ be sequences of zeroes and ones. Let $n$ be the least $j$ such that $t_{j} \neq s_{j}$. Let $m$ be the number of occurrences of the symbol 1 in the string $t_{0} t_{1} \ldots t_{m-1}=s_{0} s_{1} \ldots s_{m-1}$. If $m$ is even then define $t \prec s$ if, and only if, $t_{m}<s_{m}$. If $m$ is odd then define $t \prec s$ if, and only if, $t_{m}>s_{m}$. It is easy to show that if $x<y$ then $I_{f}(x) \prec I_{f}(y)$. A sequence $K$ is shift-maximal provided that for all $j \in \mathbb{N}, \sigma^{j}(K) \prec K$ or $\sigma^{j}(K)=K$.

Given $q \in[1,2]$, we define the tent map $T_{q}$ by

$$
T_{q}(x)= \begin{cases}q x & \text { if } x \leq 1 / 2, \\ q(1-x) & \text { if } x \geq 1 / 2 .\end{cases}
$$

We will restrict this map to its core, i.e. the interval $\left[T_{q}^{2}(1 / 2), T_{q}(1 / 2)\right]$, which is the only interval that contributes to the inverse limit space, and we will rescale this restricted map $\left.T_{q}\right|_{\left[T_{q}^{2}(1 / 2), T_{q}(1 / 2)\right]}$ to the entire interval. We will call this rescaled map the tent map core and denote it by $f_{q}:[0,1] \rightarrow[0,1]$. Notice that the critical point for $f_{q}$ is not $1 / 2$, rather it is the point $c=$ $1-1 / q$. In order to ensure that $f_{q}$ is l.e.o. we also assume that $q \in[\sqrt{2}, 2]$. Due to renormalization of tent maps when $q \in[1, \sqrt{2}]$ this is not a restriction on the topology of the inverse limit space.

Theorem 5.1 ([7, Lemma III.1.6]). Let $K$ be an infinite sequence of $0 s$ and $1 s$ that is shift-maximal and primary. Then there is a parameter $q$ in $[\sqrt{2}, 2]$ generating a tent map core $f_{q}$ with kneading sequence $K$.

Lemma 5.2 ([7, Theorem II.3.8]). Let $f$ be a unimodal map of the interval with an infinite postcritical orbit. Let $J$ be an infinite sequence of $0 \mathrm{~s}$ and $1 s$. Then there is a point $x$ in $[0,1]$ with $I_{f}(x)=J$ if, and only if, $\sigma[K(f)] \preceq \sigma^{j}(J) \preceq K(f)$ for all positive integers $j$.

For the next few lemmas we assume that $f:[0,1] \rightarrow[0,1]$ is a l.e.o. unimodal map with critical point $c$. This implies that $I_{f}$ is injective. The following lemmas are immediate consequences of the definitions or the continuity of $f$ and so we have omitted their proofs.

Lemma 5.3. Let $f:[0,1] \rightarrow[0,1]$ be unimodal and l.e.o. Let $x \in[0,1]$ be such that $\omega(x)$ is countable. For any point $y \in \omega(x)$ with $\operatorname{lt}(y)=\alpha$, there is some countable ordinal $\beta \leq \alpha$ such that if $y^{\prime} \in \omega(x)$ and $f^{k}\left(y^{\prime}\right)=y$, for some $k \in \mathbb{N}$, then $\operatorname{lt}\left(y^{\prime}\right) \geq \beta$.

Now, let $\Sigma_{f}$ be the set of allowable itineraries of $f$, i.e. $\Sigma_{f}$ is the collection of all sequences of $0 \mathrm{~s}$ and $1 \mathrm{~s}$ that are either infinite or finite and have last 
symbol $C$ with the property that $\zeta \in \Sigma_{f}$ if, and only if, there is a $y \in[0,1]$ such that $I_{f}(y)=\zeta$. Let $\zeta \in \Sigma_{f}$ and let $A$ be a finite word such that $A \sqsubseteq \zeta$. Denote the set $\left\{x \in[0,1]: A \sqsubseteq I_{f}(x)\right\}$ by $W_{A}$.

Lemma 5.4. Let $f:[0,1] \rightarrow[0,1]$ be unimodal and l.e.o. Let $\zeta \in \Sigma_{f}$ and let $A$ be a finite initial segment of $\zeta$. Then $W_{A}$ is a closed interval with precritical endpoints.

Lemma 5.5. Let $f:[0,1] \rightarrow[0,1]$ be unimodal and l.e.o. Let $x \in[0,1]$ be a point that is never mapped to the critical point. Let $A$ be a finite word such that $A \sqsubseteq I_{f}(x)$. Then $x \in W_{A}^{\circ}$. Moreover for any $x \in[0,1]$ and for all $\varepsilon>0$ there is a finite initial segment $A^{\prime} \sqsubseteq I_{f}(x)$ such that $W_{A^{\prime}} \subseteq B_{\varepsilon}(x)$.

The following lemma will be applied throughout the rest of the paper to use finite words from the itinerary of a point to determine if it is in the $\omega$-limit set of the critical point. It follows easily from the other lemmas.

Lemma 5.6. Let $f:[0,1] \rightarrow[0,1]$ be unimodal and l.e.o. Let $x \in[0,1]$ be a point that is never mapped to the critical point. Suppose that $y \in[0,1]$ is also not precritical. Then $y \in \omega(x)$ if, and only if, for all finite words $A$ satisfying $A \sqsubseteq I_{f}(y)$ there is an infinite sequence $\left(k_{i}\right)_{i \in \mathbb{N}}$ of positive integers such that $A \sqsubseteq \sigma^{k_{i}}\left[I_{f}(x)\right]$.

The following lemma is an immediate consequence of Lemma 5.6.

Lemma 5.7. Let $f:[0,1] \rightarrow[0,1]$ be unimodal and l.e.o. Let $x \in[0,1]$ and suppose that $y \in \omega(x)$. Then $y$ is isolated in $\omega(x)$ if, and only if, for every finite word $W$ that is an initial segment of $I_{f}(y)$ there is a positive integer $N$ and $t \in\{0,1\}$ such that if $m>N$ and $W$ is an initial segment of $\sigma^{m}\left[I_{f}(x)\right]$ then $W t$ is also an initial segment of $\sigma^{m}\left[I_{f}(x)\right]$.

The last lemma of the section will be useful in connecting the various levels of $\omega(c)$ with the levels of $\mathbb{F} \mathrm{d}\left(\lim _{\longleftarrow}\{[0,1], f\}\right)$.

Lemma 5.8. Let $f:[0,1] \rightarrow[0,1]$ be unimodal and l.e.o. Let $x \in[0,1]$. Suppose that $\omega(x)$ is countable. Then $f\left(L_{\alpha}^{\omega(x)}\right) \supseteq L_{\alpha}^{\omega(x)}$ for all countable ordinals $\alpha$.

Proof. Since $f$ is continuous and $f[\omega(x)]=\omega(x)$ the result follows from Lemma 3.1.

6. Examples. In this section we construct a collection of tent map cores, with critical point $c$, that have the property that, for every countable ordinal $\alpha, \operatorname{Lt}[\omega(c)]=\alpha+2$. We then demonstrate in the next section that this implies that the set $\operatorname{In}(\lim \{[0,1], f\})$ has limit type $\alpha+2$. In light of Corollary 3.6 this is the richest collection of limit types possible. 
Let $\gamma$ be a countable ordinal and $\mathcal{T}=\mathcal{T}_{\gamma}$ be the well-founded $\gamma$ tree constructed via the process outlined in Section 4 . Let $\left(N_{i}\right)_{i \in \mathbb{N}}$ be an increasing sequence of positive integers with infinite complement $M$. Let $\phi$ be a bijection from $M$ to $\mathcal{T}$, and define $\Gamma$ such that given a finite sequence $r=\left(r_{1}, \ldots, r_{n}\right)$ of terms from $M, r \in \Gamma$ if, and only if, $\phi\left(r_{i+1}\right) \lessdot \phi\left(r_{i}\right)$ for each $1 \leq i \leq n-1$. Clearly $\Gamma$ is countable, so fix some enumeration $\left(r_{i}\right)_{i \in \mathbb{N}}$ of $\Gamma$. For $r_{i} \in \Gamma$ write $r_{i}=\left(r_{1}^{i}, \ldots, r_{m_{i}}^{i}\right)$.

Starting with the words $A=1001$ and $B=101$, and given $r_{i}$ in $\Gamma$, define the finite word

$$
C_{i}=A B^{r_{1}^{i}} A B^{r_{2}^{i}} A \ldots A B^{r_{m_{i}}^{i}} A
$$

where by $W^{n}$ we mean $W^{\curlywedge}$..^ $W\left(n\right.$ times), and by $C_{0}$ we mean $A$. Define

$$
s=A A B^{N_{1}} C_{1} B^{N_{2}} C_{1} B^{N_{3}} C_{2} B^{N_{4}} C_{1} B^{N_{5}} C_{2} B^{N_{6}} C_{3} B^{N_{7}} C_{1} \ldots
$$

where the subscripts of the $C_{i}$ follow the pattern

$$
1,1,2,1,2,3,1,2,3,4,1,2,3,4,5,1,2,3,4,5,6,1,2, \ldots
$$

Proposition 6.1. $s$ is strictly shift-maximal, primary and $(101)^{\infty} \prec s$. Thus there is a parameter $q \in[\sqrt{2}, 2]$ such that $f_{q}$ is the core of a tent map with kneading sequence $s$.

Proof. Clearly $(101)^{\infty} \prec s$, and because $s$ starts with $A A$ which does not reoccur in $s, s$ is primary. Let $k \in \mathbb{N}$ and consider $\sigma^{k}(s)$. If the first symbol of $\sigma^{k}(s)$ is 0 then $\sigma^{k}(s) \prec s$. Similarly if $\sigma^{k}(s)$ starts with 11 or 101 then $\sigma^{k}(s) \prec s$. Notice there is no string of 1000 in $s$. So suppose that $\sigma^{k}(s)$ begins with $1001=A$. Recall that $s=A A \ldots$, and by definition there is not another occurrence of $A A$ in $s$. So $\sigma^{k}(s)$ must start $A B=1001101$. Clearly, $A B \ldots \prec A A \ldots$ Thus $s$ is strictly shift-maximal. By Theorem 5.1 there is a parameter $q \in[\sqrt{2}, 2]$ such that the core of the tent map $f_{q}$ has kneading sequence $s$.

Let $q$ be the parameter guaranteed by the previous lemma and consider the tent map core $f_{q}$ with kneading sequence $s$. We call tent map cores constructed via the well-founded tree construction outlined above well-founded tent map cores. Let $c$ be the critical point for this map and consider $\omega(c)$. We will show that $\operatorname{Lt}[\omega(c)]=\gamma+2$. Given $x \in[0,1]$, we denote the itinerary of $x$ under $f_{q}$ by $I(x)$.

Proposition 6.2. Let $x \in[0,1]$. Then $x \in \omega(c)$ if , and only if,

$$
I(x)= \begin{cases}\sigma^{j}\left[B^{k} C_{i} B^{\infty}\right] & \text { for some } 0 \leq j<5, k \in \mathbb{N}, i \in \mathbb{N} \cup\{0\}, \\ \sigma^{\ell}\left[B^{\infty}\right] & \text { for } 0 \leq \ell<3 .\end{cases}
$$

Proof. We begin by considering the case of $x \in[0,1]$ such that $I(x)=$ $\sigma^{\ell}\left[B^{\infty}\right]$ for some $0 \leq \ell<3$. Since for each $n \in \mathbb{N}$ there is an integer $m$ such that $N_{p}>n$ for all $p>m$, and since $B^{N_{p}}$ occurs in $I(c)$ for each $p \in \mathbb{N}$, 
the point $y \in[0,1]$ with $I(y)=B^{\infty}$ is in $\omega(c)$. Recall that $\omega(c)$ is forward invariant and $x$ is clearly a forward image of $y$, hence $x \in \omega(c)$.

Consider the case of $i=0$. Suppose $x \in[0,1]$ with $I(x)=\sigma^{j}\left[B^{k} A B^{\infty}\right]$. We will show that the point $y \in[0,1]$ with $I(y)=B^{k} A B^{\infty}$ is in $\omega(c)$. Let $\left(m_{i}\right)_{i \in \mathbb{N}}$ be a sequence of positive integers such that $r_{m_{i}}=\left(r_{1}^{m_{i}}\right)$. There is a subsequence $\left(r_{m_{i_{j}}}\right)_{j \in \mathbb{N}}$ such that $r_{1}^{m_{i_{j}}} \rightarrow \infty$ as $j \rightarrow \infty$. By definition $C_{i_{j}}$ occurs infinitely often in $I(c)$ and, for sufficiently large $j, B^{k} A$ is the tail of $C_{i_{j}}$. Hence $B^{k} A$ occurs infinitely often in $I(c)$. Since

$$
C_{i_{j}}=A B^{r_{1}^{m_{i}}} A
$$

it follows that for any integer $m, C_{i_{j}} B^{m}$ occurs infinitely often in $I(c)$. Hence $B^{k} A B^{m}$ occurs infinitely often in $I(c)$ for all $m$. Thus the point $y$ is an element of $\omega(c)$. Again, since $\omega(c)$ is forward invariant, $x \in \omega(c)$.

Assume that $x \in[0,1]$ with $I(x)=\sigma^{j}\left[B^{k} C_{i} B^{\infty}\right]$ and $i \neq 0$. Since $0 \leq$ $j<5$ and $k \geq 1$ we deduce that $I(x)$ begins with one of

(1) $B^{k} A B^{r_{1}^{i}} A=(101)^{k} 1001(101)^{r_{1}^{i}} 1001$ if $k \geq 1$,

(2) $\sigma\left[B^{k} A B^{r_{1}^{i}} A\right]=01(101)^{k-1} 1001(101)^{r_{1}^{i}} 1001$ if $k>1$,

(3) $\sigma^{2}\left[B^{k} A B^{r_{1}^{i}} A\right]=1(101)^{k-1} 1001(101)^{r_{1}^{i}} 1001$ if $k>1$,

(4) $\sigma^{3}\left[B^{k} A B^{r_{1}^{i}} A\right]=(101)^{k-1} 1001(101)^{r_{1}^{i}} 1001$ if $k>1$,

(5) $\sigma^{4}\left[B^{k} A B^{r_{1}^{i}} A\right]=01(101)^{k-2} 1001(101)^{r_{1}^{i}} 1001$ if $k>2$,

(6) $\sigma\left[B A B^{r_{1}^{i}} A\right]=011001(101)^{r_{1}^{i}} 1001=\sigma^{4}\left[B^{2} A B^{r_{1}^{i}} A\right]$,

(7) $\sigma^{2}\left[B A B^{r_{1}^{i}} A\right]=11001(101)^{r_{1}^{i}} 1001$,

(8) $\sigma^{3}\left[B A B^{r_{1}^{i}} A\right]=1001(101)^{r_{1}^{i}} 1001$,

(9) $\sigma^{4}\left[B A B^{r_{1}^{i}} A\right]=001(101)^{r_{1}^{i}} 1001$,

Hence $r_{1}^{i}$ is well-defined. Since the tail of $I(x)$ is $B^{\infty}$, and $r_{1}^{i}$ is well-defined, $C_{i}$ is also well-defined. Assume that $I(x)$ begins with $B^{k} A$. Notice that each $C_{i}$ occurs infinitely often in $I(c)$, and each occurrence of $C_{i}$ in $I(c)$ is preceded by $B$. Also notice that there is an infinite subsequence $\left(N_{u_{i}}\right)_{i \in \mathbb{N}}$ of $A$ such that infinitely many occurrences of $C_{i}$ are followed by $B^{N_{u_{i}}}$, and for each $m$ there is an occurrence of the string $C_{i} B^{N_{m}}$ in $I(c)$. Hence every initial segment of $I(x)$ occurs infinitely often in $I(c)$ and we have shown that $x \in \omega(c)$. The other cases are forward images of this case, and $\omega(c)$ is forward invariant. This establishes one direction of the proposition.

Suppose that $x \in \omega(c)$. Then either $I(x)$ contains a string of the form $A=1001$, or $\sigma(A)=001$, or it does not. In the latter case $I(x)=\sigma^{\ell}\left(B^{\infty}\right)$ for some $0 \leq \ell<3$, and we are finished. So suppose that $I(x)$ contains $A$ as a subword. If it contains exactly one occurrence of $A$ or $\sigma(A)$ then there are $k \in \mathbb{N}$ and $j<5$ such that $I(x)=\sigma^{j}\left[B^{k} A B^{\infty}\right]$ and we are finished. Suppose that $I(x)$ contains more than one occurrence of $A$. The word $A A$ 
never reoccurs in $I(c)$, so $I(x)=\sigma^{j}\left[B^{k} A B^{v_{1}} A \ldots\right]$ with $0 \leq j<5$ and $k \in \mathbb{N}$. Since $x \in \omega(c)$, the word $A B^{v_{1}} A$ or $\sigma\left[A B^{v_{1}} A\right]$ occurs infinitely often in $I(c)$, so $v_{1}=r_{1}^{i}$ for some, not necessarily unique, $i$. Continuing we can write $I(x)=\sigma^{j}\left[B^{k} A B^{v_{1}} A B^{v_{2}} \ldots A B^{v_{n}} \ldots\right]$. By the construction of $I(c)$ and the fact that $x \in \omega(c)$, the sequence $\left(v_{1}, v_{2}, \ldots\right)$ must be in $\Gamma$. Since $\mathcal{T}$ is well-founded, this sequence must be finite. Hence there is some $m_{i}$ such that $r_{i}=\left(v_{1}, \ldots, v_{m_{i}}\right)$. The only possible tail for $I(x)$ is $B^{\infty}$, since every time the word $A$ occurs in $I(x)$ it does not occur more than twice. This establishes the proposition.

Proposition 6.3. Let $x \in \omega(c)$ with $I(x)=\sigma^{j}\left[B^{k} C_{i} B^{\infty}\right]$ for some $0 \leq j<5$ and $k, i \in \mathbb{N}$. Then $\operatorname{lt}(x)=\operatorname{ht}_{\mathcal{T}}\left[\phi\left(r_{m_{i}}^{i}\right)\right]$.

Proof. Let $x \in \omega(c)$ with $I(x)=\sigma^{j}\left[B^{k} C_{i} B^{\infty}\right]$ for some $0 \leq j<5$ and $k, n \in \mathbb{N}$. Assume that $\operatorname{ht}_{\mathcal{T}}\left[\phi\left(r_{m_{i}}^{i}\right)\right]=0$. Then there is no $\ell$ such that $r_{\ell}$ contains $r_{m_{i}}^{i}$ as anything but the last entry. Hence for every $N$, there is an integer $M$ such that if $C_{i} \sqsubseteq \sigma^{n}[I(c)]$ then $C_{i} B^{M} \sqsubseteq \sigma^{n}[I(c)]$ for all $n>N$. Thus by Lemma 5.7, $x$ is isolated in $\omega(c)$ and $\operatorname{lt}(x)=0$.

Let $\zeta<\gamma$ and assume the assertion is true for all $\beta<\zeta$. Also assume that $\operatorname{ht}_{\mathcal{T}}\left[\phi\left(r_{m_{i}}^{i}\right)\right]=\zeta$. We first consider the case of $\zeta$ a limit ordinal. Let $\left(\zeta_{q}\right)_{q \in \mathbb{N}}$ be a sequence of ordinals converging to $\zeta$, and for a fixed $q$ and for each positive integer $v$, let $r_{j_{q, v}}$ be such that $\operatorname{ht}_{\mathcal{T}}\left[\phi\left(r_{m_{j_{q, v}}}^{j_{q, v}}\right)\right]=\zeta_{q}$ and $r_{m_{j_{q, v}}-1}^{j_{q, v}}=r_{m_{i}}^{i}$. By the construction of $\mathcal{T}$, such an $r_{j_{q, v}}$ can be defined for each $q, v \in \mathbb{N}$. We also know, by the construction of $\mathcal{T}$, that $r_{m_{j_{q, v}}}^{j_{q, v}} \rightarrow \infty$ as $v \rightarrow \infty$. Hence the points $y_{q, v} \in \omega(c)$ with $I\left(y_{q, v}\right)=\sigma^{j}\left[B^{k} C_{j_{q, v}} B^{\infty}\right]$ have $\operatorname{lt}\left(y_{q, v}\right)=\zeta_{q}$, and since $r_{m_{j_{q, v}}}^{j_{q, v}} \rightarrow \infty$ we see that $y_{q, v} \rightarrow x$ as $v \rightarrow \infty$. Thus $\operatorname{lt}(x)>\zeta_{q}$. Since this is true for each $q, \operatorname{lt}(x) \geq \zeta$. A similar argument shows that $\operatorname{lt}(x) \geq \zeta$ in the case that $\zeta$ is a successor ordinal.

To prove that $\operatorname{lt}(x)=\zeta$, let $z \in \omega(c), z \neq x$, be such that $I(z)=$ $\sigma^{t}\left[B^{u} C_{v} B^{\infty}\right]$ where $t<5, u, v \in \mathbb{N}$ and $\operatorname{ht}_{\mathcal{T}}\left[\phi\left(r_{m_{v}}^{v}\right)\right]=\zeta$. Then $\operatorname{lt}(z) \geq \zeta$. Clearly there exists a positive number $\delta_{1}$ so that if $C_{v}$ has a different number of occurrences of $A$ than $C_{i}$ does, then $z \notin B_{\delta_{0}}(x)$. So assume that $C_{v}$ has the same number of occurrences of $A$ that $C_{i}$ has. This is the same as assuming that $r_{v}$ has the same number of terms as $r_{i}$. Let $\delta_{1}$ be small enough so that if $y \in B_{\delta_{1}}(x)$ then $I(y)$ agrees with $I(x)$ past the occurrence of $C_{i}$ in $I(x)$. This implies that $z \notin B_{\delta}(x)$ where $\delta=\min \left\{\delta_{0}, \delta_{1}\right\}$. Since this is true for any $z \neq x$ with $\operatorname{lt}(z) \geq \zeta$, we infer that $x$ is not the limit of points of limit type $\zeta$. Thus $\operatorname{lt}(x) \leq \zeta$, and combining this with the previous we see that $\operatorname{lt}(x)=\zeta$.

Proposition 6.4. Let $x \in \omega(c)$ with $I(x)=\sigma^{j}\left[B^{k} A B^{\infty}\right]$ with $0 \leq j<5$ and $k \in \mathbb{N}$. Then $\operatorname{lt}(x)=\gamma$. 
Proof. Let $x \in \omega(c)$ be such that $I(x)=\sigma^{j}\left[B^{k} A B^{\infty}\right]$ for some $0 \leq j<5$ and $k \in \mathbb{N}$. Let $\beta<\gamma$. We will show that $\operatorname{lt}(x)>\beta$. Let $\left(r_{i}\right)_{i \in \mathbb{N}}$ be a sequence such that $\operatorname{ht}_{\mathcal{T}}\left[\phi\left(r_{m_{i}}^{i}\right)\right]=\beta$ for all $i \in \mathbb{N}$ and $r_{1}^{i} \neq r_{1}^{\ell}$ for all $\ell, i \in \mathbb{N}$. The existence of such a sequence is guaranteed by the construction of $\mathcal{T}$, and it is easy to see that $r_{1}^{\ell} \rightarrow \infty$ as $\ell \rightarrow \infty$. Let $y_{i} \in \omega(c)$ be the point such that $I\left(y_{i}\right)=\sigma^{j}\left[B^{k} C_{i} B^{\infty}\right]$ for the same $j$ and $k$ specified earlier. Then $\operatorname{lt}\left(y_{i}\right)=\beta$ for all $i$ and $y_{i} \rightarrow x$ as $i \rightarrow \infty$. Thus $\operatorname{lt}(x)>\beta$ for all $\beta<\gamma$. Hence $\operatorname{lt}(x) \geq \gamma$.

Since each point $y \in \omega(c)$ with $I(y)=\sigma^{t}\left[B^{u} C_{v} B^{\infty}\right]$ for $0 \leq t<5$, $u, v \in \mathbb{N}$ has $\operatorname{lt}(y)<\gamma$, the only points in $\omega(c)$ with limit type greater than or equal to $\gamma$ are points $z \in \omega(c)$ with $I(z)=\sigma^{t}\left[B^{u} A B^{\infty}\right]$ or $I(z)=\sigma^{\ell}\left[B^{\infty}\right]$ where $0 \leq t<5, u \in \mathbb{N}$ and $0 \leq \ell<3$. Clearly there is a positive number $\varepsilon$ such that no such $z \neq x$ is in $B_{\varepsilon}(x)$. Hence $x$ is not a limit of points with limit type $\gamma$, so $\operatorname{lt}(x) \leq \gamma$. Combining this with the previous yields $\operatorname{lt}(x)=\gamma$.

Proposition 6.5. Let $x_{\ell} \in \omega(c)$ with $I\left(x_{\ell}\right)=\sigma^{\ell}\left[B^{\infty}\right]$ with $0 \leq \ell<3$. Then $\operatorname{lt}\left(x_{\ell}\right)=\gamma+1$.

Proof. Let $x_{\ell} \in \omega(c)$ with $I\left(x_{\ell}\right)=\sigma^{\ell}\left[B^{\infty}\right]$ for $\ell<3$. Let $\left(k_{i}\right)_{i \in \mathbb{N}}$ be an increasing sequence of integers. Let $z_{i} \in \omega(c)$ be such that $I\left(z_{i}\right)=$ $\sigma^{\ell}\left[B^{k_{i}} A B^{\infty}\right]$. By Proposition 6.4, $\operatorname{lt}\left(z_{i}\right)=\gamma$ and clearly $z_{i} \rightarrow x_{\ell}$ as $i \rightarrow \infty$. Thus $\operatorname{lt}\left(x_{\ell}\right) \geq \gamma+1$. There are only three possible points, $x_{0}, x_{1}, x_{2}$, with limit type greater than $\gamma+1$. Hence there are no points in $\omega(c)$ with limit type $\gamma+2$. Thus $\operatorname{lt}\left(x_{\ell}\right)=\gamma+1$.

Corollary 6.6. $\operatorname{Lt}[\omega(c)]=\gamma+2$.

Proof. This follows immediately from the previous propositions.

As a result of this construction we have the following theorem.

Theorem 6.7. Let $\gamma$ be a countable ordinal and let $n \in \mathbb{N}$. Then there is a tent map core, $f:[0,1] \rightarrow[0,1]$, with critical point $c$ such that

(1) $\operatorname{Lt}[\omega(c)]=\gamma+2$,

(2) $\left|L_{\gamma+1}^{\omega(c)}\right|=n$.

Proof. By the examples constructed earlier in this section, for any countable ordinal $\gamma$, there is a tent map core, $f$, such that $\operatorname{Lt}[\omega(c)]=\gamma+2$. Moreover, given $n \in \mathbb{N}$, we could have chosen a different finite word $B$, corresponding to a period $n$ orbit, and also a word $A=10^{j} 1$ that would guarantee that the sequence built is primary and shift-maximal.

7. Folding points in inverse limit spaces. In this section we use the collection of examples constructed in the previous section to show that there is a nonhyperbolic invariant set $X$ with a countable collection of inhomogeneities of $X, \operatorname{In}(X)$, displaying any topological structure not precluded 
by Corollary 3.6. The spaces we build are just the inverse limits of the various tent map cores $f_{q}$ constructed in the previous section. We will denote the inverse limit of $f$ by $X_{f}$ and the inverse limit of $f_{q}$ by $X_{q}$. Since these spaces are inverse limits of continuous maps on intervals, they are chainable continua, and so $\operatorname{In}\left(X_{q}\right)=\mathbb{F} \mathrm{d}\left(X_{q}\right)$. In [16] we prove the following theorem:

TheOREM 7.1. Let $f:[0,1] \rightarrow[0,1]$ be unimodal with critical point $c$. Let $x \in X_{f}$. Then $x \in \mathbb{F} \mathrm{d}\left(X_{f}\right)$ if, and only if, for every $n \in \mathbb{N}, \pi_{n}(x) \in \omega(c)$.

We extend that theorem to the following corollary that allows us to analyze the structure of the sets $L_{\alpha}^{\mathbb{I n}\left(X_{f}\right)}=L_{\alpha}^{\mathbb{F d}\left(X_{f}\right)}$.

Corollary 7.2. Let $f:[0,1] \rightarrow[0,1]$ be unimodal with critical point $c$ such that $f$ is l.e.o. Let $x \in X_{f}$. If $\alpha$ is the least ordinal such that $\pi_{n}(x) \in L_{\alpha}^{\omega(c)}$ for infinitely many $n \in \mathbb{N}$, then $\operatorname{lt}(x) \geq \alpha$.

Proof. Let $x \in \mathbb{F} \mathrm{d}\left(X_{f}\right)$. For each $n$, let $\beta_{n}$ be an ordinal such that $\pi_{n}(x)=x_{n} \in L_{\beta_{n}}^{\omega(c)}$. Then $\left(\beta_{n}\right)_{n \in \mathbb{N}}$ is a nonincreasing sequence of ordinals. Hence it only contains finitely many ordinals. Let $\gamma$ be the least. Since the sequence is nonincreasing, there exists an integer $m$ such that $\beta_{i}=\gamma$ for all $i \geq m$. So the ordinal mentioned in the statement of the corollary is well-defined for any point in $\mathbb{F} \mathrm{d}\left(X_{f}\right)$. Moreover, if $\alpha$ is the least ordinal $\beta$ such that $x_{n} \in L_{\beta}^{\omega(c)}$ for infinitely many $n$, then in fact $\alpha$ is the only ordinal $\beta$ such that $x_{n} \in L_{\beta}^{\omega(c)}$ for infinitely many $n$.

The case of $\alpha=0$ is obvious. We proceed inductively. Let $\alpha>0$ be an ordinal and assume the corollary is true for all $\beta<\alpha$. Let $x \in X_{f}$ with $x \in \mathbb{F} \mathrm{d}\left(X_{f}\right)$ be such that $\alpha$ is the ordinal such that for all $n>M$ we have $x_{n} \in L_{\alpha}^{\omega(c)}$ for some $m \in \mathbb{N}$. Then clearly for any $\beta<\alpha$ any neighborhood containing $x$ will contain points $y$ with the property that $y_{n} \in L_{\beta}^{\omega(c)}$ for infinitely many $n$. Hence any neighborhood of $x$ will contain points $y$ with $\operatorname{lt}(y) \geq \beta$. Thus $x \in \mathbb{F} \mathrm{d}\left(X_{f}\right)^{(\zeta)}$, the $\zeta$ th iterated CantorBendixson derivative of $\mathbb{F} \mathrm{d}\left(X_{f}\right)$, for some $\zeta \geq \alpha$. Hence $\operatorname{lt}(x) \geq \alpha$.

In order to strengthen the statement of Corollary 7.2, we will use the precise structure of the well-founded tent map cores to show that for the point $x$ mentioned in Corollary 7.2 we have $\operatorname{lt}(x)=\alpha$. To that end we prove the following lemmas.

LEMMA 7.3. Let $f$ be a well-founded tent map core with critical point $c$. Let $q \in M$. There is a finite sequence of allowable words $r_{i} \in \Gamma$ such that $r_{m_{i}}^{i}=q$. Moreover, for any pair $r$, s of such allowable words, either $r$ is a tail of $s$ or $s$ is a tail of $r$.

Proof. Recall that a word $v=\left(v_{1}, \ldots, v_{m}\right)$ is allowable provided that $\phi\left(v_{i}\right) \lessdot \phi\left(v_{i-1}\right)$. By the construction of $\mathcal{T}$, given any $d \in \mathbb{N}^{\prime}$ there is exactly 
one $d_{-1} \in \mathbb{N}^{\prime}$ such that $\phi(d) \lessdot \phi\left(d_{-1}\right)$. This fact combined with the fact that $\mathcal{T}$ is well-founded proves the lemma.

Let $x_{0} \in \omega(c)$. Call an infinite sequence $\left(x_{0}, x_{-1}, x_{-2}, \ldots\right)$ permitted provided $f\left(x_{-i}\right)=x_{-(i-1)}$ and $x_{-i} \in \omega(c)$ for each $i \in \mathbb{N}$. Assume that $\alpha<\omega_{1}$ and $x_{0} \in L_{\alpha}^{\omega(c)}$. Call a permitted sequence $\left(x_{0}, x_{-1}, x_{-2}, \ldots\right) \alpha$-permitted provided that $x_{-i} \in L_{\alpha}^{\omega(c)}$ for all $i \in \mathbb{N}$.

LemmA 7.4. Let $x_{0} \in \omega(c)$, and let $\left(x_{0}, x_{-1}, x_{-2}, \ldots\right)$ be a permitted sequence. There is an ordinal $\alpha<\omega_{1}$ and a positive integer $j$ such that the sequence $\left(x_{-j}, x_{-(j+1)}, \ldots\right)$ is $\alpha$-permitted.

Proof. There is no infinite decreasing sequence of ordinals and $\operatorname{lt}(x) \leq$ $\operatorname{lt}(f(x))$.

Lemma 7.5. Let $\alpha<\omega_{1}$, and let $z \in L_{\alpha}^{\omega(c)}$. There is at least one and no more than finitely many $\alpha$-permitted sequences $\left(x_{0}, x_{-1}, x_{-2}, \ldots\right)$ with $x_{0}=z$.

Proof. Since $f\left(L_{\alpha}^{\omega(c)}\right) \supseteq L_{\alpha}^{\omega(c)}$, given such a point $z$ there is an $\alpha$ permitted sequence $\left(x_{0}, x_{-1}, x_{-2}, \ldots\right)$ with $x_{0}=z$. Let $\left(y_{0}, y_{-1}, y_{-2}, \ldots\right)$ be some $\alpha$-permitted sequence.

By Proposition 6.2,

$$
I(z)= \begin{cases}\sigma^{j}\left[B^{k} C_{i} B^{\infty}\right] & \text { for some } 0 \leq j<5, k \in \mathbb{N}, i \in \mathbb{N} \cup\{0\}, \\ \sigma^{\ell}\left[B^{\infty}\right] & \text { for } 0 \leq \ell<3 .\end{cases}
$$

We handle the first case first. Assume that $I(z)=\sigma^{j}\left[B^{k} C_{i} B^{\infty}\right]$ for some $0 \leq j<5$ and $k, i \in \mathbb{N}$. Then $\alpha=\operatorname{lt}(z)=\operatorname{ht}_{\mathcal{T}}\left[\phi\left(r_{m_{i}}^{i}\right)\right]$, by Proposition 6.3. By Lemma 7.3 there are only finitely many possible words $C_{u}$ with $r_{m_{u}}^{u}=r_{m_{i}}^{i}$. For each of these strings $C_{u}$ longer than $C_{i}$, there is a point $y \in L_{\alpha}^{\omega(c)}$ such that $f^{n}(y)=z$ for some $n \in \mathbb{N}$ with $I(y)=\sigma^{s}\left[B^{t} C_{u} B^{\infty}\right]$ for some $0 \leq s<5$ and $t \in \mathbb{N}$. Also, if $w \in L_{\alpha}^{\omega(c)}$ is such that $f^{n}(w)=z$ for some $n \in \mathbb{N}$, then $I(w)=\sigma^{s}\left[B^{t} C_{u} B^{\infty}\right]$ for some $0 \leq s<5, t \in \mathbb{N}$, and for one of the finitely many $u$ 's. Thus for each of the finitely many $u$ 's there is a single $\alpha$-permitted sequence $\left(y_{0}, y_{-1}, y_{-2}, \ldots\right)$ with $y_{0}=z$, and for some fixed positive integer $M_{u}$ and all $m \geq M_{u}$ have $I\left(y_{-m}\right)=\sigma^{s}\left[B^{t} C_{u} B^{\infty}\right]$. This proves the lemma for this first case.

If instead $I(z)=\sigma^{\ell}\left[B^{\infty}\right]$ for some $0 \leq \ell<3$, then $\operatorname{lt}(z)=\gamma+2$ and there are exactly three (or $n$ if we constructed the kneading sequence of $f$ with a different word $B$ ) points with that limit type. So there are only three $(\gamma+2)$-permitted sequences. 
Lemma 7.6. Let $x \in \lim _{\longleftarrow}\{[0,1], f\}$ have $\pi_{n}(x)=x_{n} \in \omega(c)$ for all $n \in \mathbb{N}$. Let $\alpha<\omega_{1}$ be such that $x_{j} \in L_{\alpha}^{\omega(c)}$ for all $j \geq J$. Then there are finitely many points $z \in \lim \{[0,1], f\}$ such that $z_{n} \in \omega(c)$ for all $n \in \mathbb{N}$ and $z_{J}=x_{J}$ and $z_{j} \in L_{\alpha}^{\omega(c)}$ for all $j \geq J$.

Proof. This follows from the fact that each such $z$ corresponds to an $\alpha$-permitted sequence $\left(y_{0}, y_{-1}, y_{-2}, \ldots\right)$ with $z_{J}=y_{0}=x_{J}$. Since there are only finitely many such sequences, there are only finitely many points $z \in \lim _{\longleftarrow}\{[0,1], f\}$.

Theorem 7.7. Let $x \in \varliminf_{\lfloor}\{[0,1], f\}$ have $\pi_{n}(x)=x_{n} \in \omega(c)$ for all $n \in \mathbb{N}$. Let $\alpha<\omega_{1}$ be such that $x_{j} \in L_{\alpha}^{\omega(c)}$ for all $j \geq J$. Then $x \in$ $L_{\alpha}^{\mathbb{F} d\left(\lim _{\longleftarrow}\{[0,1], f\}\right)}$.

Proof. By Proposition 7.2 we know that $\operatorname{lt}(x) \geq \alpha$. By Lemma 7.6, there are only finitely many points $z$ with $z_{J}=x_{J}$ and $z_{j} \in L_{\alpha}^{\omega(c)}$ for all $j \geq J$. Thus $\operatorname{lt}(x)=\alpha$.

Proposition 7.8. Let $f:[0,1] \rightarrow[0,1]$ be a well-founded tent map core with critical point $c$. Assume that $\operatorname{Lt}[\omega(c)]=\gamma+2$ for some ordinal $\gamma$, and let $\left|L_{\gamma+1}^{\omega(c)}\right|=n$. Then

(1) $\operatorname{Lt}\left[\mathbb{F} \mathrm{d}\left(X_{f}\right)\right]=\gamma+2$,

(2) $\left|L_{\gamma+1}^{\mathbb{F d}\left(X_{f}\right)}\right|=n$,

(3) $\left|L_{\beta}^{\mathbb{F d}\left(X_{f}\right)}\right|=\aleph_{0}$ for all $\beta<\gamma+1$.

Proof. By Lemma 5.8, $f\left(L_{\beta}^{\omega(c)}\right) \supseteq L_{\beta}^{\omega(c)}$ for all $\beta<\gamma+2$. Hence for each $x_{0}$ in $L_{\beta}^{\omega(c)}$, we can build a sequence $\left(x_{i}\right)_{i \in \mathbb{N}}$ of preimages such that $f\left(x_{i+1}\right)=x_{i}$ and $x_{i} \in L_{\beta}^{\omega(c)}$ for all $i$. Thus $x=\left(x_{0}, x_{1}, x_{2} \ldots\right) \in X_{f}$, and by Proposition 7.7, $x \in L_{\beta}^{\mathbb{F d}\left(X_{f}\right)}$ because $x$ always projects into $L_{\beta}^{\omega(c)}$.

Since the top level of $\omega(c)$ in the Cantor-Bendixson decomposition partitions into finitely many periodic orbits, it follows that for any well-founded tent map there are a finite number of periodic points that generate the points in the inverse limit space that have the most topologically "complicated" neighborhoods.

Let $X$ be a chainable continuum with $\mathbb{F d}(X)$ countable. We define the folding spectrum of $X, \operatorname{FS}(X)$, to be the ordered pair $(\alpha, n)$, where $\alpha=$ $\operatorname{Lt}[\mathbb{F} \mathrm{d}(X)]$ and $n=\left|L_{\alpha-1}^{\mathbb{F d}(X)}\right|$. Note that $\operatorname{FS}(X)$ is topological. This observation together with the examples of the well-founded tent map cores constructed in the previous section leads to the following theorem. 
THEOREM 7.9. There are uncountably many nonhomeomorphic tent map inverse limit spaces.

In fact, we have shown that there are $\omega_{1}$ nonhomeomorphic tent map inverse limits. This theorem should be contrasted with [3, Corollary 2] in which Barge and Diamond demonstrate that there are uncountably many, in fact $\mathfrak{c}$ many, nonhomeomorphic inverse limits spaces of unimodal maps. However, the maps they consider are infinitely renormalizable unimodal maps, such as logistic maps. The maps we consider here are not infinitely renormalizable, being locally eventually onto. Also Brucks and Bruin have shown in [6] that there are uncountably many tent map inverse limits. However, in their paper they consider spaces with a Cantor set of folding points and complicated subcontinua (at least more complicated than simply arcs). In our case we have only countably many folding points and every proper subcontinuum is an arc. In a forthcoming paper [9], we prove that there are nonhomeomorphic tent map inverse limits, each with a Cantor set of folding points and with every proper subcontinuum being an arc.

8. Counting parameters. In this section we demonstrate that, although the construction in $\S 6$ is quite delicate and particular, given a possible folding spectrum $(\alpha, n)$, the collection of parameters $s$ corresponding to a well-founded tent map core $f_{s}$ that generates an inverse limit with folding spectrum precisely $(\alpha, n)$ is a dense subset of $[\sqrt{2}, 2]$ and it has cardinality $\mathfrak{c}$.

We use two well-known facts regarding tent maps (cf. [7] and [8, pp. 235 $\& 238])$. The first fact is that the parameters $q \in[\sqrt{2}, 2]$ that generate tent map cores $f_{q}$ with periodic, preperiodic, or prefixed critical points are dense in $[\sqrt{2}, 2]$. We also use the fact that if $\varepsilon>0$ and $q \in[\sqrt{2}, 2]$ then there is a finite word $W \sqsubset K_{f_{q}}$ such that if $r \in[\sqrt{2}, 2]$ and $K_{f_{r}}$ is a kneading sequence such that $W \sqsubset K_{f_{r}}$ then $r \in(q-\varepsilon, q+\varepsilon) \cap[\sqrt{2}, 2]$.

TheOREM 8.1. Let $q \in[\sqrt{2}, 2]$ be such that $f_{q}$ is a well-founded tent map core with $\mathrm{FS}\left(\lim _{\longleftarrow}\left\{[0,1], f_{q}\right\}\right)=(\alpha, n)$ for some countable ordinal $\alpha$ and positive integer $n$. The set $\left\{r \in[\sqrt{2}, 2]: \operatorname{FS}\left(\lim _{\longleftarrow}\left\{[0,1], f_{r}\right\}\right)=(\alpha, n)\right\}$ has cardinality $\mathfrak{c}$.

Proof. Let $q \in[\sqrt{2}, 2]$ be such that $f_{q}$ is a well-founded tent map core with $\operatorname{FS}\left(\varliminf_{\longleftarrow}\left\{[0,1], f_{q}\right\}\right)=(\alpha, n)$ for some countable ordinal $\alpha$ and positive integer $n$. Let $K$ denote the kneading sequence of $f_{q}$, and let $c$ denote the critical point of $f_{q}$. Since $\omega(c)$ is countable, it contains a periodic point and its orbit $\left\{x_{0}, x_{1}, \ldots, x_{n-1}\right\}$. Let $B$ be a finite word made up of symbols from $\{0,1\}$ such that $I_{f_{q}}\left(x_{0}\right)=B^{\infty}$. Since every initial segment of $I_{f_{q}}$ occurs infinitely often in $K$, we have an increasing sequence $\left(n_{i}\right)_{i \in \mathbb{N}}$ of positive integers such that $B^{n_{i}} W_{i}$ is an initial segment of $\sigma^{k_{i}}(K)$ for some positive integer $k_{i}$ and word $W_{i}$ that does not have $B$ as an initial segment. The 
words $W_{i}$ can be chosen in such a way that we can write $K$ as

$$
K=W_{0} B^{n_{1}} W_{1} B^{n_{2}} W_{3} B^{n_{4}} \ldots
$$

Let $\tau=\left(t_{i}\right)_{i \in \mathbb{N}}$ be a sequence such that $t_{i} \in\{0,1\}$ for all $i \in \mathbb{N}$. Define $K_{\tau}$ by

$$
K_{\tau}=W_{0} B^{n_{1}+t_{1}} W_{1} B^{n_{2}+t_{2}} W_{2} B^{n_{3}+t_{3}} \ldots
$$

Clearly if we choose $W_{0}$ to be long enough, $K_{\tau}$ will be primary and shiftmaximal. So there is a number $r \in[\sqrt{2}, 2]$ such that $f_{r}$ has kneading sequence $K_{\tau}$. It is easy to see that since $n_{i} \rightarrow \infty$ as $i \rightarrow \infty$ the critical $\omega$-limit set for $f_{r}$ is homeomorphic to the critical $\omega$-limit set for $f_{q}$ and $f_{r}$ is a well-founded tent map core. By Proposition 7.8 we conclude that $\mathrm{FS}\left(\varliminf_{\lim }\left\{[0,1], f_{q}\right\}\right)=\mathrm{FS}\left(\varliminf_{\lim }\left\{[0,1], f_{r}\right\}\right)$. Notice that there are $\mathfrak{c}$ possible choices for $\tau$, each resulting in a different $K_{\tau}$ and hence a different parameter $r$. This establishes the theorem.

TheOREM 8.2. Let $q \in[\sqrt{2}, 2]$ be such that $f_{q}$ is a well-founded tent map core with $\mathrm{FS}\left(\varliminf_{\mathfrak{T}}\left\{[0,1], f_{q}\right\}\right)=(\alpha, n)$ for some countable ordinal $\alpha$ and positive integer $n$. The set $\left\{r \in[\sqrt{2}, 2]: \operatorname{FS}\left(\varliminf_{\lim }\left\{[0,1], f_{r}\right\}\right)=(\alpha, n)\right\}$ is dense in $[\sqrt{2}, 2]$.

Proof. Write $\alpha=\beta+2$. Let $z \in[\sqrt{2}, 2]$, and let $\varepsilon>0$. It is well-known that the set of parameters that give rise to tent maps with a preperiodic critical point that gets mapped to a period $n$ orbit is dense in $[\sqrt{2}, 2]$ (see [8, Lemma 7.3]). Let $q_{0}$ be such a parameter in $B_{\varepsilon / 2}(z)$. Let $B_{0}$ be a word of length $n$ in symbols 0,1 such that the kneading sequence of the map $f_{q_{0}}$ is $K_{f_{q_{0}}}=10^{j} 1 W B_{0}^{\infty}$ where $j$ is a positive integer and $W$ is a finite (or empty) word in 0,1 . We can choose a finite word $V$ such that $10^{j} 1 W \sqsubseteq V \sqsubset K_{f_{q_{0}}}$ and if $r \in[\sqrt{2}, 2]$ with $V \sqsubset K_{f_{r}}$ then $r \in B_{\varepsilon / 2}\left(q_{0}\right) \subseteq B_{\varepsilon}(z)$.

We can construct a sequence $K$ in the manner described in Section 6 using a well-founded $\alpha-2=\beta$-tree, the word $B_{0}$ in place of $B, 10^{j} 1$ as $A$, but with $K$ beginning with $V$ instead of $A A \ldots$. Since $K_{q_{0}}$ is shift-maximal and primary, we can ensure that $K$ is also shift-maximal and primary. Then there is an $r \in B_{\varepsilon / 2}\left(q_{0}\right) \subset B_{\varepsilon}(z)$ such that $K_{f_{r}}=K$. Denote the critical point of $f_{r}$ by $c_{r}$. Then by our construction $\operatorname{Lt}\left[\omega\left(c_{r}\right)\right]=\alpha,\left|L_{\alpha-1}^{\omega\left(c_{r}\right)}\right|=n$ and $f_{r}$ is a well-founded tent map core. Hence, by Proposition 7.8, FS $\left(\lim _{\longleftarrow}\left\{[0,1], f_{r}\right\}\right)$ $=(\alpha, n)$.

\section{References}

[1] J. E. Anderson and I. F. Putnam, Topological invariants for substitution tilings and their associated $C^{*}$-algebras, Ergodic Theory Dynam. Systems 18 (1998), 509-537.

[2] M. Barge, K. Brucks, and B. Diamond, Self-similarity in inverse limit spaces of the tent family, Proc. Amer. Math. Soc. 124 (1996), 3563-3570. 
[3] M. Barge and B. Diamond, Inverse limit spaces of infinitely renormalizable maps, Topology Appl. 83 (1998), 103-108.

[4] - - - A complete invariant for the topology of one-dimensional substitution tiling spaces, Ergodic Theory Dynam. Systems 21 (2001), 1333-1358.

[5] M. Barge, J. Jacklitch, and G. Vago, Homeomorphisms of one-dimensional inverse limits with applications to substitution tilings, unstable manifolds, and tent maps, in: Geometry and Topology in Dynamics (Winston-Salem, NC, 1998/San Antonio, TX, 1999), Amer. Math. Soc., Providence, RI, 1999, 1-15.

[6] K. Brucks and H. Bruin, Subcontinua of inverse limit spaces of unimodal maps, Fund. Math. 160 (1999), 219-246.

[7] P. Collet and J.-P. Eckmann, Iterated Maps on the Interval as Dynamical Systems, Birkhäuser, Boston, MA, 1980.

[8] E. M. Coven, I. Kan, and J. A. Yorke, Pseudo-orbit shadowing in the family of tent maps, Trans. Amer. Math. Soc. 308 (1988), 227-241.

[9] C. Good and B. E. Raines, Continuum many tent map inverse limits with homeomorphic postcritical omega-limit sets, Fund. Math. 191 (2006), 1-21.

[10] J. Guckenheimer and P. Holmes, Nonlinear Oscillations, Dynamical Systems, and Bifurcations of Vector Fields, Appl. Math. Sci. 42, Springer, New York, 1990. Revised and corrected reprint of the 1983 original.

[11] W. T. Ingram, Inverse Limits, Sociedad Matemática Mexicana, México, 2000.

[12] W. T. Ingram and W. S. Mahavier, Interesting dynamics and inverse limits in a family of one-dimensional maps, Amer. Math. Monthly 111 (2004), 198-215.

[13] A. S. Kechris, Classical Descriptive Set Theory, Grad. Texts in Math. 156, Springer, New York, 1995.

[14] J. Kennedy and J. T. Rogers, Jr, Orbits of the pseudocircle, Trans. Amer. Math. Soc. 296 (1986), 327-340.

[15] B. Raines, One-dimensional dynamics and inverse limits, D. Phil. thesis, Oxford Univ., 2002.

[16] - Inhomogeneities in nonhyperbolic one-dimensional invariant sets, Fund. Math. 182 (2004), 241-268.

[17] L. Sadun and R. F. Williams, Tiling spaces are Cantor set fiber bundles, Ergodic Theory Dynam. Systems 23 (2003), 307-316.

[18] R. F. Williams, One-dimensional non-wandering sets, Topology 6 (1967), 473-487.

[19] - Expanding attractors, Inst. Hautes Études Sci. Publ. Math. 43 (1974), 169-203.

[20] I. Yi, Canonical symbolic dynamics for one-dimensional generalized solenoids, Trans. Amer. Math. Soc. 353 (2001), 3741-3767.

School of Mathematics and Statistics

University of Birmingham

Birmingham, B15 2TT, UK

E-mail: c.good@bham.ac.uk

Mathematical Institute

University of Oxford

Oxford OX1 3LB, UK

E-mail: knight@maths.ox.ac.uk

Department of Mathematics

Baylor University

Waco, TX 76798-7328, U.S.A.

E-mail: brian_raines@baylor.edu 